\title{
Comunicación

\section{Everett M. Rogers (1931-2004) y la investigación Latinoamericana de la comunicación}

RAÚL FUENTES NAVARRO ${ }^{1}$

En este artículo se analiza la influencia del enfoque de la difusión de innovaciones en la investigación latinoamericana de la comunicación, especialmente en la década de los años sesenta, en el contexto de la modernización y el impulso al desarrollo. Como un recuerdo del papel desempeñado por Everett M. Rogers, recientemente fallecido, en esa etapa de la historia del campo con su trabajo en Colombia, se incorporan algunos testimonios personales registrados en una entrevista sostenida con él a fines de 1994.

PALABRAS ClaVE: modernización, comunicación para el desarrollo, difusión de innovaciones, historia de la investigación, Colombia
This contribution analyzes the influence exerted by the diffusion of innovations approach on Latin American communication research, particularly in the sixties, in the context of modernization and developing of societies. Remembering the late Everett $M$. Rogers and his role over this historic period through his work in Colombia, some remarks from him are included, taken from an interview recorded in 1994.

Key words:modernization, communication for development, diffusion of innovations, history of investigation, Colombia.

1 Profesor investigador del Departamento de Estudios de la Comunicación Social de la Universidad de Guadalajara y del Departamento de Estudios Socioculturales del ITESO. Miembro del Sistema Nacional de Investigadores, nivel II, y de la Academia Mexicana de Ciencias.

Correo electrónico: raul@iteso.mx 
El 21 de octubre de 2004, en Albuquerque, Nuevo México, murió Everett M. Rogers, uno de los investigadores estadounidenses más influyentes y respetados en el campo de la comunicación en buena parte del mundo, incluyendo América Latina. Nacido 73 años antes en una granja de Iowa, estudió agricultura, sociología y estadística en la universidad estatal de su estado, donde se doctoró en 1957. En 1962 publicó la primera versión de su teoría de la difusión de innovaciones, que le dio fama mundial y que lo ubicó como uno de los fundadores de la "comunicación para el desarrollo" y de la "comunicación internacional". Fue profesor en seis universidades estadounidenses durante su prolífica carrera de casi cinco décadas y trabajó como experto en diversos países de África, Asia y América Latina.

A fines de 1994 estuvo en Guadalajara, como conferencista invitado durante la Feria Internacional del Libro. En esa ocasión, Enrique Sánchez Ruiz (quien años atrás fue alumno de Rogers en Stanford) y Raúl Fuentes Navarro sostuvimos una charla con él, que quedó grabada pero que hasta ahora fue transcrita y editada. ${ }^{2}$ En esa charla, informal y cordial como era el estilo personal de Rogers, comentamos algunos de los episodios en que fue protagonista directo en el campo de la investigación latinoamericana de la comunicación. Se trataba de conocer su reconstrucción personal, a la distancia, de las circunstancias en que sucedieron ciertos hechos, obligatoriamente registrados en la historia del campo.

En aquel 1994, Rogers publicó Una historia del estudio de la comunicación, un acercamiento biográfico (Rogers, 1994). En el prefacio de esa obra declaró que:

Un historiador del estudio de la comunicación podría organizar esta cronología por épocas históricas, por filosofías dominantes (por ejemplo, el progresismo), por las tecnologías comunicacionales de estudio (cine, radio, televisión), o por otros factores contextuales. Mi elección es darle sentido a la historia del estudio de la comunicación sobre la base de las personas, mediante la historiografía biográfica (Rogers, 1994:X-XI).

Además de las fuentes documentales y bibliográficas, Rogers utilizó sobre todo las entrevistas de historia oral para construir su obra, en la

2 Por la transcripción de las grabaciones, un agradecimiento a Ruby Sheets. 
que analiza el "mito de los cuatro padres fundadores" (Lasswell, Hovland, Lazarsfeld y Lewin) y propone en su lugar a Wilbur Schramm como el verdadero fundador del campo de estudios de la comunicación. ${ }^{3}$

En este artículo se pretende, mediante una emulación de los métodos favoritos de Rogers para documentar la historia del campo, incorporar algunos testimonios personales del investigador a una revisión de los aportes académicos formales de su trabajo a la investigación de la comunicación en América Latina, especialmente los ubicados en Colombia en los años sesenta. Con ello se busca actualizar la conciencia, entre los investigadores de la comunicación en ejercicio y en formación, sobre los temas y los enfoques, los sujetos y los contextos que influyeron y que, de distintas maneras, siguen presentes aunque los marcos dominantes de referencia hayan cambiado en la configuración histórica de los estudios comunicacionales.

\section{EL CONTEXTO: COMUNICACIÓN PARA}

\section{LA MODERNIZACIÓN DE AMÉRICA LATINA ${ }^{4}$}

En los años sesenta se extendió por toda América Latina el afán "modernizador" y "desarrollista", formulado por la sociología del desarrollo estadounidense, que incluía el impulso de investigaciones sobre la estructura económico-social y sobre actitudes y opiniones de sectores significativos del "sistema de estratificación social". Para los teóricos del desarrollo, los países de la región lo alcanzarían detectando y controlando los obstáculos internos, implementando un "plan de desarrollo", acelerando el aporte de capital extranjero y estimulando un nuevo tipo de personalidad caracterizada por los valores predominantes en los países centrales. La investigación social, hasta entonces incipientemente desarrollada en América Latina, adoptó no sólo las metas propuestas, sino también los valores predominantes de la ciencia norteamericana de la posguerra:

3 En 1997, con Steven Chaffee, Rogers editó otra obra con y sobre las contribuciones de Schramm a la historia del campo en Estados Unidos (Schramm, 1997).

4 Esta sección está basada en una versión anterior, más amplia, publicada en Fuentes (1992). 
Objetivismo, empirismo estadístico, neutralidad valorativa, asepsia ideológica, énfasis metodológico. La investigación científica era tal en tanto que fuera lo más neutral y objetiva posible en la conquista de su meta principal: la obtención de un conocimiento empíricamente fundado (Murga y Boils, 1979:18).

Proveniente tanto de esa sociología como de la psicología social, la "ciencia de la comunicación humana" se había constituido en Estados Unidos en torno a lo que ha sido llamado el "paradigma dominante". Este modelo científico debe mucho al aporte, entre otros, del especialista en la investigación de la propaganda política y la psicología, Harold Lasswell, quien en 1948 estableció que:

Una manera conveniente de describir un acto de comunicación es la que surge de la contestación a las siguientes preguntas: ¿Quién dice qué en qué canal a quién y con qué efecto? El estudio científico del proceso de comunicación tiende a concentrarse en una u otra de tales preguntas. Los eruditos que estudian el "quién", el comunicador, contemplan los factores que inician y guían el acto de la comunicación. Llamamos a esta subdivisión del campo de investigación análisis de control. Los especialistas que enfocan el "dice qué" hacen análisis de contenido. Aquéllos que contemplan principalmente la radio, la prensa, las películas y otros canales de comunicación, están haciendo análisis de medios. Cuando la preocupación primordial se centra en las personas a las que llegan los medios, hablamos de análisis de audiencia. Y si lo que interesa es el impacto sobre las audiencias, el problema es el del análisis de los efectos (Lasswell, 1985:51).

Sobre bases como éstas, y las aportadas por otros investigadores desde los años veinte (Moragas, 1981; Saperas, 1985; Rogers, 1994), se desarrolló en Estados Unidos la Mass Communication Research, en un entorno que le disponía todas las condiciones favorables, pues contó con financiamientos y apoyos de los más altos centros políticos, militares y de espionaje estadounidenses, además de los recursos necesarios para la aplicación de conocimientos y la prueba de hipótesis cada vez más refinadas en el inmejorable laboratorio de la sociedad estadounidense. Pero también, cada vez más, en otros países del hemisferio, en el 
contexto de la guerra fría. Es desde esta posición y este "paradigma dominante" desde donde Wilbur Schramm podía tranquilamente afirmar a principios de los sesenta que:

La investigación sobre comunicación en los Estados Unidos es cuantitativa, más que especulativa. Quienes la practican... son investigadores del comportamiento: tratan de encontrar algo acerca de por qué los humanos se comportan en la forma en que lo hacen y cómo puede la comunicación hacer posible que vivan juntos más feliz y productivamente. Por lo tanto, no es de sorprender que numerosos investigadores de la comunicación se hayan ocupado últimamente del problema de la forma en que las naciones del mundo pueden comunicarse eficientemente y cómo puede ayudarles la comunicación a comprenderse mejor entre sí y a vivir en paz (Schramm, 1961).

La instalación y operación funcional de sistemas de comunicación de masas fue un aspecto paulatinamente incorporado al perfil de la modernización de los países "subdesarrollados". Aunque desde muchos años antes en Estados Unidos estos sistemas eran reconocidos como "clave fundamental" del desarrollo, en América Latina, no fue sino hasta la década de los sesenta, prácticamente con el inicio de la expansión de la televisión, cuando se comenzó a considerar y a investigar con cierta profundidad la comunicación. Enrique Sánchez Ruiz, en su estudio sobre la modernización (1986), sintetiza críticamente el proceso de adopción de la ciencia de la comunicación estadounidense en América Latina:

Parece haber acuerdo en que el trabajo de Daniel Lerner, The Passing of Traditional Society, publicado por primera vez en 1958, fue el principal punto de partida de la visión optimista sobre el papel de la comunicación de masas en la modernización (...). El modelo original de la modernización de Lerner, que fue seguido por una gran cantidad de estudios, partió de la observación de ciertas correlaciones entre algunas variables en países del Medio Oriente, de donde se generalizó para intentar explicar cómo ocurrirían los procesos de desarrollo nacional entendidos como procesos de "modernización": se suponía que la industrialización tendía a elevar la urbanización; ésta, a su vez, elevaría la alfabetización, seguida por un incremento en la exposición de la población a los medios masivos de comu- 
nicación. El alfabetismo y la exposición a los medios producirían en los individuos "empatía" (la habilidad psicológica de ponerse uno en lugar de otro) o "movilidad psicológica", lo que finalmente tendería a aumentar la participación política y económica.

Éste era un modelo causal, lineal, elegante y optimista que, debido a su simplicidad, atrajo la atención de investigadores dentro del campo de la comunicación y la modernización. Aún más, Lerner (1963) propuso posteriormente toda una "teoría de la modernización basada en la comunicación", a partir de su trabajo previo, la cual fue tomada seriamente y aun expandida por algunos investigadores (...).

Es curioso notar que durante la década en que, a partir de la publicación del influyente libro de Joseph Klapper (1960) sobre los efectos de la comunicación de masas, los investigadores de la comunicación dentro de Estados Unidos dudaban que los medios fueran una influencia potente real para el cambio, los investigadores norteamericanos que trabajaban en contextos subdesarrollados se encontraban "comprometidos con la visión de que tales medios podrían y habrían de producir cambios profundos" (Krippendorff, 1979:75). El trabajo de Wilbur Schramm (1964), Medios masivos y desarrollo nacional, continuó y extendió la presuposición teórica de que los medios participaban en el desarrollo como agentes de cambio (Sánchez Ruiz, 1986:22-23).

El Centro Internacional de Estudios Superiores de Periodismo (después, Comunicación) para América Latina (CIESPAL) fue uno de los principales centros difusores e impulsores de estos estudios sobre comunicación y modernización en América Latina desde su fundación en Quito en 1959. Entre sus primeras tareas, además de la formación de profesores para las escuelas de periodismo, estuvo la organización de cursos regionales con los más destacados investigadores estadounidenses y europeos en el campo de la comunicación para el desarrollo y la edición de traducciones de sus principales obras. Para el impulso a la investigación, CIESPAL extendió dos modelos: la metodología del francés Jacques Kayser para el análisis morfológico y de contenido de la prensa, y las técnicas norteamericanas de análisis de audiencias y de 
efectos de los medios masivos. José Marques de Melo contextualiza el papel de CIESPAL:

En los países del Tercer Mundo, el incremento de la investigación en comunicación es el resultado de la acción desarrollada por la UNESCO para lograr la ampliación de las redes nacionales de comunicación colectiva. Su motivación es democratizar las oportunidades educacionales; supone que los medios o vehículos electrónicos (radio y televisión) posibilitarán la alfabetización en masa, la educación continuada de las minorías poblacionales, a bajo costo. Dentro de este esfuerzo educativo, los países pobres importaron tecnología, sistemas gerenciales, modelos científicos, y tuvieron que formar recursos humanos para el manejo de los bienes adquiridos. CIESPAL surge en la coyuntura latinoamericana como una iniciativa de la UNESCO para diseminar matrices destinadas a la preparación de profesionales para los medios de comunicación colectiva que atiendan a las nuevas exigencias socio-culturales (Marques de Melo, 1984:5).

Entre los textos más representativos de la importación del "paradigma dominante" en el estudio de la comunicación y su aplicación a los programas de modernización en América Latina, puede citarse el titulado "Desarrollo de la comunicación y desarrollo económico" de Wilbur Schramm, entonces director del Instituto de Investigación en Comunicación de la Universidad Stanford, traducido y editado en 1965 por el Instituto Interamericano de Ciencias Agrícolas de la OEA, con sede en San José, Costa Rica. La breve introducción describe el enfoque de su contenido:

Conforme las naciones avanzan de los patrones de la sociedad tradicional hacia los de la sociedad industrial moderna, tienen lugar desarrollos espectaculares en su comunicación. Desde un cierto punto de vista, estos cambios en la comunicación son causados por los avances económicos, sociales y políticos que son parte del crecimiento nacional. Desde otro, sin embargo, los desarrollos en comunicación están entre los principales factores e impulsores de los otros cambios. Es propósito de este estudio explorar esta interacción y buscar una base para comprenderla (Schramm, 1965:1). 
A partir de ahí, el trabajo se divide en cuatro partes. La primera, "Comunicación en sistemas nacionales", explica las interacciones postuladas entre la comunicación y el desarrollo, desde un punto de vista teórico general. En la segunda, "La comunicación como impulsora", toma como base las propuestas de Rostow (1960) y Lerner (1958) para establecer seis "condiciones previas" para el desarrollo nacional en las cuales debe contribuir la comunicación:

A. La comunicación debe emplearse para contribuir al sentimiento de nacionalidad.

B. La comunicación debe emplearse como la voz del planeamiento nacional.

C. La comunicación debe emplearse para ayudar a enseñar las destrezas necesarias.

D. La comunicación debe usarse para ayudar a ampliar el mercado efectivo.

E. Conforme el plan se desarrolla, la comunicación debe usarse en ayudar a la gente a representar sus nuevos papeles.

F. La comunicación debe usarse para preparar a la gente a representar su papel como nación entre naciones (Schramm, 1965:6-11).

La tercera parte corresponde a la interacción recíproca: "La economía como impulsora" y en ella se subraya que "la estrategia económica del desarrollo de la comunicación no se puede dividir en una estrategia para la educación y otra para la información; la estrategia debe ser una sola" (Schramm, 1965:19). Por último, en la cuarta, "Algunas consecuencias políticas implícitas" de la interacción de la economía con la comunicación, Schramm señala, entre otras cosas, que:

El poder está donde está el control. No es casi necesario recordarle este hecho a un científico de la política, o a un político, pero en un país en desarrollo, donde los canales de comunicación son en su mayor parte de poco alcance y personales, el control de los canales de largo alcance llega a ser dramáticamente importante (Schramm, 1965:21).

Hasta los años sesenta, la mayoría de los estudios empíricos sobre la comunicación en América Latina fueron investigaciones realizadas, o al menos orientadas, por estadounidenses. Así como para las décadas 
anteriores Rita Atwood había señalado la parcialidad etnocéntrica y la aplicación de modelos causales en los trabajos sobre América Latina publicados en las revistas especializadas en Estados Unidos, a los sesenta les llama la "década de la diversificación", aunque las características predominantes de los estudios siguieron siendo, según su análisis, las mismas: "Puede decirse que los datos y las conclusiones que se presentan en estos artículos reflejan más la realidad del investigador-observador que la del actor-sujeto, y que diferencias más importantes pueden separar a ambas" (Atwood, 1980:10-14).

Pero dentro de la notable expansión que, a pesar de todos los sesgos y limitaciones, experimentó la investigación de la comunicación en América Latina en la "primera década del desarrollo", como llamó la ONU a los sesenta, y en el contexto precisamente de los afanes de modernización, debe prestarse una especial atención a la difusión de innovaciones, cuya singularidad reside, según Marques de Melo, "en haber penetrado en las áreas rurales de América Latina, hasta entonces poco exploradas por cientistas sociales y revelado una serie de facetas inéditas de las culturas regionales" (Marques de Melo, 1984:5-6).

En 1976, la Oficina Regional para América Latina del Centro Internacional de Investigaciones para el Desarrollo (CIID), entidad descentralizada del Gobierno de Canadá, publicó en su sede de Bogotá una Bibliografía sobre investigaciones en comunicación para el desarrollo rural en América Latina preparada por Luis Ramiro Beltrán y otros, donde se anotan las referencias de 490 estudios "no urbanos y no foráneos realizados mediante el empleo, en algún grado y forma, del método científico" (Beltrán, Isaza y Ramírez, 1976). El más antiguo de estos estudios está fechado en 1955 y la recopilación llega hasta 1975. Esta obra nos permite ver que la investigación para el desarrollo rural se concentró en tres países: Colombia, Brasil y México, en los cuales en conjunto se realizaron dos de cada tres de los estudios compilados. ${ }^{5}$ La mayor parte de tales investigaciones estu-

5 Para el caso de Brasil, José Marques de Melo recopiló en 1978, bajo el título Comunicación, modernización y difusión de innovaciones en Brasil, 186 referencias de estudios realizados tanto por estadounidenses (algunos de ellos conocidos como "brasileñistas") como por brasileños, entre ellos sesenta tesis de posgrado presentadas sobre todo en las universidades de Wisconsin, 
vieron basadas en el enfoque de la difusión de innovaciones, impulsado por Everett Rogers. La obra clásica de Rogers, Difusión de Innovaciones, publicada originalmente en 1962, estableció una línea de investigación cuyo modelo, en palabras del propio autor, consiste en lo siguiente:

Este modelo describe el proceso mediante el cual una innovación (definida como una idea práctica u objetivo percibido como nuevo por un individuo) es comunicada por medio de ciertos canales a través del tiempo a miembros de un sistema social. El modelo clásico especifica: 1) Los estudios en el proceso decisión-innovación y la importancia relativa de varios canales en cada uno de los estudios; 2) la forma en la cual las características percibidas de las innovaciones afectan su tasa de adopción; 3) las características y el comportamiento de los adoptadores "tempranos" y "tardíos"; 4) el rol de los líderes de opinión en la difusión de innovaciones; y 5) los factores que intervienen en el éxito relativo de los agentes de cambio.

(...) El modelo clásico se originó a partir de los estudios efectuados por los sociólogos de los años cuarenta sobre la amplia utilización de innovaciones agrícolas (como el maíz híbrido). Actualmente, después de dos mil investi-

Michigan State e Illinois, sobre Brasil específicamente, y cincuenta referencias más de estudios generales o comparativos que incluían a Brasil. Una situación muy similar, con el desarrollo de programas e investigaciones prácticamente idénticas que las realizadas en Colombia y en Brasil, se registró en México. Desde los trabajos de Alfonso Ruanova (1956; 1958), Gregorio Martínez (1960; 1962; 1970), Jesús Martínez Reding (1963; 1965), Leobardo Jiménez $(1963 ; 1967)$ y otros agrónomos egresados del Departamento de Periodismo Agrícola de la Universidad de Wisconsin y adscritos a la Rama de Divulgación Agrícola del Colegio de Posgraduados de Chapingo, hasta las dos Reuniones Nacionales de Comunicación Social en el Medio Rural (1978 y 1979), organizadas por el Centro Nacional de Productividad (CeNaPro), la contribución mexicana a la investigación sobre la difusión de innovaciones agrícolas es también considerable. Quizá el llamado "Plan Puebla" sea, por su extensión y amplia documentación de la investigación, el proyecto mexicano más importante en cuanto a comunicación y desarrollo rural. Algunos de los estudios referidos a él son los de Jairo Cano (1971), Díaz Cisneros y Felstenhausen (1972) y Delbert T. Myren (1974). 
gaciones y treinta y tres años, el enfoque de difusión todavía lleva consigo el sello de su origen intelectual, a pesar de que las bases de investigación se han ampliado para incluir innovaciones tales como medios anticonceptivos y la atención de una variedad de sociólogos (Rogers, 1973:74-75).

Esta descripción del desarrollo del enfoque es perfectamente documentable: Rogers y muchos de sus seguidores fueron modificando los postulados, métodos, estrategias y técnicas de investigación conforme se iban acumulando experiencias, no sólo en el campo del desarrollo rural, sino en muchos otros, y no sólo en Estados Unidos o América Latina, sino en prácticamente el mundo entero. ${ }^{6}$

\section{RECUERDOS DE UNA ÉPOCA PASADA}

Por razones diversas, en Colombia se concentraron, desde los años cincuenta, muchos de los esfuerzos pioneros de la investigación latinoamericana para el desarrollo rural. Anzola y Cooper (1985) destacan, por un lado, la creación en 1947 de Acción Cultural Popular (ACPO) y su programa de educación rural a través de las Escuelas Radiofónicas y, por otro lado, a la institución del "Frente Nacional", un pacto entre partidos para la gobernabilidad, en 1958. El contexto general en los sesenta lo resumen así:

Fruto de la política sectorial de los sucesivos gobiernos surgen varias instituciones descentralizadas a cuyo amparo florecerá la investigación en comunicación durante este periodo y el siguiente (1970-79). Es de particular relevancia la estrategia de cambio social que se impulsa para el sector

6 Una mención especial en el área de la difusión de innovaciones y la comunicación para el desarrollo rural en América Latina debe dedicársele a Juan Díaz Bordenave, cuyos estudios pioneros en el nordeste brasileño (1964; 1966) fueron después ampliados hasta llevarlo a la capacitación de expertos latinoamericanos $(1972,1974)$ y a una continuada presencia e incansable labor, incorporando a su experiencia y formación en la tradición estadounidense los aportes críticos de Paulo Freire, raíz de muchos de los cuestionamientos que pusieron en crisis en los setenta a la investigación latinoamericana en comunicación para el desarrollo. 
rural, encarnada en la propuesta de reforma agraria (finalmente fallida) y en los dos institutos creados para apoyarla: el Instituto Colombiano de Reforma Agraria (INCORA) y el Instituto Colombiano Agropecuario (ICA).

Este último, fundado en 1963, cuenta desde ese momento con un Departamento de Ciencias Sociales encargado de la investigación de apoyo a los programas de extensión: es el inicio de los estudios en comunicación para el desarrollo rural, y particularmente de la línea difusionista apoyada por fundaciones y universidades de los Estados Unidos, que reflejan las políticas de ese país representadas en la Alianza para el Progreso.

La influencia norteamericana se da también a través de los programas de formación a nivel superior en ese país, que acogen a un número pequeño pero significativo de expertos en comunicación que, al regresar al país, forman el núcleo básico de investigadores particularmente en el ICA; a lo cual se añade la presencia e incidencia académica de algunos profesores norteamericanos que se vinculan a universidades colombianas (Anzola y Cooper, 1985:XVI).

Everett Rogers fue uno de esos “profesores norteamericanos". A la distancia, en 1994, Rogers consideraba que el desarrollo del campo de la comunicación en Latinoamérica es "un proceso activo", que no consiste sólo "en recibir y aplicar teorías y métodos de otros países, o de mezclarlos":

Se trata de un híbrido, pues el campo que se desarrolla aquí yo creo que será diferente, de maneras muy importantes, tanto de un punto de vista europeo como de uno norteamericano. Diferente de un acercamiento empírico. Diferente de un acercamiento crítico. No sé, no estoy seguro exactamente de qué forma tomará en América Latina, pero estoy muy interesado en ello. ${ }^{7}$

No obstante, Rogers reconoció estar "en realidad unos quince años desactualizado" pues, "por alguna razón, mi vida parece haberse ido

7 A partir de aquí, las citas textuales presentadas en itálicas corresponden a la transcripción (traducida al español por Raúl Fuentes Navarro) de las expresiones de Everett M. Rogers en la entrevista grabada el 30 de noviembre de 1994 en instalaciones de la Universidad de Guadalajara. 
hacia la India y África en la última década, así que no he tenido tanto contacto con Latinoamérica". Entendía, o justificaba, este alejamiento como parte de un proceso más amplio:

Creo que ahora hay menos contactos entre Estados Unidos y, ciertamente, México, pero probablemente toda América Latina. Alguna vez -y esto pudo haber sido, digamos, entre 1960 y 1980 o un poco antes-por veinte años hubo mucho contacto en ambas direcciones. Muchos académicos estadounidenses de comunicaciones venían a América Latina, frecuentemente a enseñar o a hacer investigación. Yo fui uno de ésos. También mucha gente de América Latina, la mayoría mexicanos, iban a estudiar en universidades norteamericanas. Así que había mucho intercambio en ambas direcciones. No era exactamente un intercambio de iguales en mi opinión, pero hay mucho menos ahora. Si contáramos el número de académicos de la comunicación que cruzan la frontera en cualquier dirección, serían menos de los que eran. No sé si esto es malo; yo creo que probablemente es bueno.

La estancia de Rogers en Colombia y su influencia desde ahí en la investigación latinoamericana de la comunicación para el desarrollo han sido documentadas desde distintos ángulos, aunque su propia reconstrucción aporta algunos datos interesantes sobre las circunstancias en las que se dio, y que permiten detectar también una influencia de América Latina en él:

Lo primero que supe realmente sobre Colombia fue lo que leí en un artículo de investigación de Fals Borda y Paul Deutschmann. Deutschmann fue un pionero de los estudios de comunicación en Michigan State. Murió en 1962 de un ataque cardiaco. Era el tipo de persona que no vive para el mañana. Se quedaba trabajando toda la noche. Estaba enamorado de los datos y de la investigación. Un hombre maravilloso, pero que no era de esperarse que estuviera por ahí demasiado tiempo. Él estaba muy interesado por América Latina, así que se fue en 1959 a dirigir un centro de investigación en Costa Rica. Era básicamente un centro para hacer investigación de la comunicación, financiado por la Fundación Rockefeller. Y se suponía que él viajara por toda América Latina y colaborara con latinoamericanos para realizar estudios en sus países. Yo nunca entendí por 
qué la Rockefeller aportó ese dinero pero lo hizo, y nunca pareció que hubiera que retribuirlo en algo. Ciertamente, no influyó en nada sobre lo que se estudiaba.

Deutschmann fue a Bogotá y se asoció con Orlando Fals Borda. Fals Borda había obtenido su doctorado en sociología en Minnesota y su tesis doctoral fue un estudio de un pueblito andino, llamado Saucío, a sesenta millas de Bogotá. Hizo una encuesta y una observación participante. Fals Borda vivió varios meses al año en Saucío, e hizo ahí investigación-acción, aunque creo que entonces todavía no se le llamaba así. Promovió una cooperativa de mujeres e introdujo varias innovaciones agrícolas en la aldea. Su interés central era el cambio social.

Deutschmann y Fals Borda tenían mucho en común. Congeniaron y decidieron hacer un estudio de difusión en Saucío. Este estudio fue realizado por estudiantes e investigadores de la Facultad de Sociología de la Universidad Nacional de Colombia, y era un estudio de difusión agrícola como muchos que se hacían entonces. Yo estaba haciendo un trabajo similar en Ohio, como profesor de Ohio State. Conocía a Deutschmann y al leer lo que hacía en Saucío pude ver que había muchas similitudes con lo que yo estaba haciendo. Habría que ver los informes publicados... pero su último capítulo era una comparación de sus resultados en Saucío con los míos en Ohio.

El trabajo de Deutschmann y Fals Borda (quien después sería uno de los impulsores de la "investigación participativa" latinoamericana, fuertemente impregnada de marxismo) es, efectivamente, un estudio de la difusión de seis innovaciones agrícolas en la aldea colombiana de Saucío y su comparación con investigaciones similares en Estados Unidos. Las conclusiones son que el fenómeno se da de manera similar, por lo que el concepto de innovación es igualmente aplicable, a pesar de algunas diferencias en el proceso (Deutschmann y Fals-Borda, 1962). Hay dos informes más que, junto a éste, representan de la mejor manera la investigación de la comunicación para el desarrollo rural en Colombia, y en ambos participó Everett Rogers (con Bonilla y con Herzog). Son parte de la múltiple documentación producida por el proyecto que en 1964 presentó Rogers a la Agency for International Development 
(AID), para ser realizado en cuatro años, bajo el título "Difusión de innovaciones en sociedades rurales" (Rogers, 1964), del cual vale la pena transcribir algunos párrafos:

En tanto que se ha realizado mucha investigación sobre la difusión y adopción de prácticas agrícolas en los Estados Unidos (una revisión reciente de literatura incluye más de 600 publicaciones), sólo unas 40 investigaciones sobre este tópico se han realizado en sociedades en desarrollo. De éstas, pocas tienen el nivel de precisión planeado para este estudio. Se reconoce que hay factores específicos asociados con la adopción de nuevas ideas agrícolas en las culturas tradicionales. Los hallazgos norteamericanos no pueden ser aplicados en otros países sin una prueba apropiada. Un contexto cultural diferente cuenta mucho para que ciertos hallazgos no puedan sostenerse y otros deban ser considerablemente modificados.

Por ejemplo, una generalización de varios investigadores norteamericanos es que la comunicación masiva es el factor más importante para crear conciencia de la innovación, pero la comunicación personal con amigos y vecinos es la más importante para convencer a los agricultores de adoptar la innovación. Lo que no se sabe hasta ahora es la validez de esta generalización en sociedades tradicionales, donde los medios masivos o no existen o son muy escasos.

A diferencia de los Estados Unidos, las decisiones de adoptar o rechazar una innovación agrícola en culturas tradicionales pueden ser tomadas, $\mathrm{o}$ al menos muy influidas, por la familia entera más que por el agricultor individual. Por ello es especialmente importante considerar a la familia campesina y también la influencia del pueblo o comunidad cuando se investiga la adopción de tecnología agrícola.

Los resultados de la investigación propuesta serán de utilidad en todo tipo de programas de desarrollo, como los programas de asistencia técnica de la AID, de las Naciones Unidas, de la Alianza para el Progreso, los Cuerpos de Paz de los Estados Unidos, las fundaciones y agencias privadas y los programas nacionales de desarrollo de la comunidad y de servicios de extensión. La investigación contribuirá a la comprensión teórica del proceso 
de desarrollo económico y social nacional. Los programas de cambio pueden ser planeados más efectivamente cuando se basan en una comprensión más adecuada de la difusión y de la adopción de prácticas agrícolas entre gente relativamente tradicional. La meta eventual de este tipo de investigación es acortar el tiempo requerido para que la tecnología agrícola alcance un uso amplio entre las familias campesinas de las naciones tradicionales. (...)

Esta investigación busca acelerar la adopción de tecnología agrícola explicando por qué los programas de cambio en la producción agrícola son relativamente exitosos o no exitosos en aldeas rurales de sociedades en desarrollo. (...) Más específicamente, el presente estudio enfoca tres clases de objetivos: el conocimiento útil para los agentes de cambio, los métodos útiles para investigaciones posteriores y el reforzamiento de las instituciones participantes tanto de los Estados Unidos como de los países huéspedes (Rogers, 1964:1-5).

Los criterios para seleccionar los países latinoamericanos, africanos y asiáticos donde se realizaría el proyecto, incluyeron el que el país tuviera un programa de acción para incrementar la producción agrícola, que hubiera un programa de asistencia técnica de la AID y contratos de ésta con universidades tanto estadounidenses como locales. Entre los países probables se mencionaban India, Turquía y Pakistán en Asia; Colombia y Brasil en América Latina; Nigeria y Kenya en Africa.

Aunque el proyecto presentado por Rogers a la AID en 1964 incluía el trabajo de campo en varios países y continentes, su reconstrucción del contacto con Colombia tiene algunos elementos adicionales de interés, detrás de su narración anecdótica:

Así que yo conocía a Deutschmann, pero no a Fals Borda, quien debe haber sabido de mí a través de Deutschmann. Pero en el verano de 1961, en St. Louis, en el congreso de la American Sociological Association, un colega mío de Ohio State y yo presentamos una ponencia sobre la investigación de difusión que estábamos haciendo. Yo leí la ponencia y entonces un joven y apuesto latinoamericano se acercó a estrechar mi mano y a decir en perfecto inglés que le había gustado mi trabajo. Dejó su tarjeta 
que, de un lado, en letras doradas, decía: Orlando Fals Borda, decano... y del otro, escrito con pluma, "si quieres un trago de buen whisky, ven a la habitación 631".

A mi colega le gustaba el whisky, así que fuimos a la habitación 631 del hotel. Ahi estaba Fals Borda, efectivamente con una botella de buen whisky en la mano. Le dije: "Tú debes ser el Fals Borda que colaboró con Deutschmann en su estudio de difusión”. El dijo "sí”, y también: "Quiero que vengas a Colombia a hacer estudios de difusión y a enseñar a mis estudiantes a hacerlos". A mi colega y a mí nos pareció una locura. Yo tenía fuertes intereses internacionales, pero siempre quise ir a hacer estudios en la India. Ya había leído libros sobre India. Estaba enamorado de la India. Y no estaba enamorado de América Latina. La única palabra en español que sabía, creo, era "adiós”. De manera que nada estaba más lejos de mi intención que una beca Fulbright. Pero Fals Borda dijo que pensaba poder arreglarlo si yo podía ir. Como yo no podría el año siguiente, a mi colega, que estaba terminando su tesis doctoral, le entusiasmó la idea, aunque tampoco sabía español. Fals Borda le dijo: "Pues más vale que comiences ya a estudiar español”.

Al regresar a Ohio, no pensamos que pasaría nada, pero al llegar nos encontramos una carta de Fals Borda escrita desde Washington. Había hecho escala en Washington volando de St. Louis a Bogotá. Y había visitado la Comisión Fulbright y nos envió las formas de solicitud. En su nota decía que estaba aceptado en principio que fuéramos los profesores Fulbright en su facultad los dos años siguientes. Primero mi colega, luego yo. Comenzamos a estudiar español. Mi colega fue a Colombia en 1962-1963 y yo en 19631964. Por varios años hubo una cadena de gente de Ohio State y de otros lados que nos siguió. Sea como sea, hice un estudio de difusión en comunidades rurales colombianas y eso fue un paso adelante.

De la documentación sobre la difusión de innovaciones agrícolas en Colombia, que es muy extensa y detallada, pueden tomarse dos ejemplos: el informe de la investigación de Rogers y Bonilla de Ramos (1965) consiste en la descripción sistemática de "nuestros varios intentos para predecir la adopción de una innovación (la siembra de hortalizas) en tres 
pueblos campesinos de los Andes colombianos, usando tres métodos diferentes de predicción". Para el estudio se seleccionaron como variables independientes la empatía, la cosmopoliticidad, las normas del cambio social, la exposición a los medios masivos y el alfabetismo de los sujetos. El informe de Rogers y Herzog (1966) sobre el "alfabetismo funcional entre campesinos colombianos", estudio también realizado en cinco comunidades campesinas de los Andes, da cuenta de que se encontraron altas correlaciones entre el alfabetismo funcional y el auto-concepto de alfabetismo y los años de escolaridad. Pero se encontró también que el alfabetismo funcional estaba relacionado con la exposición a los medios masivos, que era más característico de los niños que de los adultos, que estaba asociado con la empatía, la innovatividad doméstica y agrícola, con la motivación de logro, el tamaño de la granja, los viajes a centros urbanos, el conocimiento político y el liderazgo de opinión sociométrico.

Más allá de los resultados empíricos es claro que, al estudiar el "cambio social", se implicaba también la necesidad del "cambio científico", y Rogers narraba con bastante detalle cómo se fue percatando de esta situación:

Durante el año que estuve en Colombia, Fals Borda seguía estando muy comprometido con los estudios en que se colectaban datos empíricos cuantitativos, pero estaba comenzando a tener algunas dudas. Yo también empezaba a tenerlas. Impartí dos cursos de metodología: uno para estudiantes de tercer año, otro para los de cuarto año. Los estudiantes participaban también conmigo en la recolección de datos de los estudios que hacía. Así empecé a ver lo que no podría medir con encuestas y análisis de datos cuantitativos... Varios académicos de la facultad estaban haciendo estudios etnográficos y daban cursos de antropología.

Yo mantuve estrecho contacto con la comunidad y con Fals Borda y con los estudiantes que había enseñado. Varios de ellos fueron a Michigan State a estudiar y yo les ayudé con su trabajo de campo en Colombia. Fue durante esos años que muchas cosas cambiaron. (...) Se trataba de un país que no funcionaba muy bien en esa época. La estructura social mostraba inequidades básicas muy profundas y esos asuntos eran los que yo 
no conocía cuando fui a Colombia. Éste fue un periodo de cuestionamiento para mí (...) y para Orlando Fals Borda. Yo creo que el hecho clave, sin embargo, fue la muerte de Camilo Torres.

Conocí a Torres muy bien. Estaba enseñando en la Facultad en el mismo tiempo que yo. Era hijo de una familia colombiana muy rica. Y como en cualquier familia colombiana de esa época, un hijo debía ser ingeniero, otro hijo doctor y otro sacerdote. Como era de una familia rica, fue enviado a la Universidad de Lovaina en Bélgica, donde obtuvo su doctorado en Sociología. Cuando regresó, era el capellán de la universidad. Volvió más radicalizado que cuando se fue. Comenzó a cuestionar muchas cosas. Eventualmente fue despedido de su capellanía porque era demasiado radical. Marchó a la cabeza de una manifestación estudiantil. El rector de la universidad protestó ante el administrador de la educación, que protestó frente al Cardenal, y ése fue el fin de Camilo Torres como capellán.

Él había estado dando un curso de sociología de la religión y los profesores y los estudiantes lo estimaban, pues era un profesor muy efectivo. Así se convirtió en docente de tiempo completo en la Facultad. Eso fue durante el año que yo estuve ahí. Dos años después, digamos que fue perdiendo la fe en el establecimiento. Eventualmente, como se sabe, se unió a la guerrilla en las montañas y lo mataron. Se convirtió en un símbolo muy importante para todos los jóvenes colombianos. Era un amigo cercano de Fals Borda. Yo pienso que su radicalización, y en último término su muerte, tuvieron un efecto en todos nosotros, pero uno mayor en Fals Borda. Fue un hecho crucial: hizo que Fals Borda renunciara a la decanatura de la Facultad. Abrió un instituto independiente, desconectado de la universidad, hizo otro tipo diferente de investigación y ejerció una influencia muy diferente. También terminó nuestra cercanía. (...) Fue un periodo de radicalización de las ciencias sociales de todo tipo en Colombia.

La investigación latinoamericana en ciencias sociales en general se radicalizó en el discurso y en sus articulaciones sociales en los años setenta. Los debates sobre la "cientificidad" o la imposición "imperialista" de modelos ideológicos predominaron en los círculos de estudio de la comunicación, dentro y fuera de las universidades. Uno de los 
blancos preferidos de estas críticas fue el trabajo de Rogers, por lo que parecía representar. Se hablaba ya no de la difusión de innovaciones, sino de su "infusión”. Pero, para él,

Cualquiera que quisiera criticar cualquier cosa que yo hubiera hecho o representado o propuesto, académicamente, yo creo que era perfectamente apropiado y correcto, y eso nunca me molestó. Me interesó mucho como fenómeno que los puntos de vista de las ciencias sociales en Colombia hubieran cambiado tanto en diez años. Comencé a cuestionar los estudios de modernización después de haber hecho al menos un estudio de ésos. Creo que las críticas me hicieron pensar que no haría otros estudios como ésos y generalmente he tratado de hacer diferentes tipos de investigación.

Everett Rogers es recordado, especialmente por quienes fueron sus alumnos, como un investigador abierto siempre a las críticas a su trabajo y a la incorporación constante de los debates suscitados por él; en este sentido, como un ejemplo de honestidad intelectual. ${ }^{8}$

\section{CRISIS Y CRÍTICA DEL PARADIGMA DOMINANTE}

Así, después de más de diez años de un intensivo y extenso esfuerzo de investigación sobre la comunicación y el desarrollo en América Latina y en otras regiones del mundo, en que los medios masivos fueron considerados "multiplicadores mágicos" y se estudiaron sus efectos en la innovación de las prácticas agrícolas, educativas, políticas y de salud pública en muchos países, Everett Rogers sintetizó en 1976 un consenso creciente entre los seguidores de la tradición estadounidense en una frase: "El decepcionante desempeño del paradigma dominante durante la década pasada lleva a considerar varias concepciones alternativas de la comunicación en el desarrollo" (Rogers, 1976:100). En el número de Communication Research dedicado a las "Perspectivas críticas sobre comunicación y de-

8 Arvind Singhal, actualmente profesor en la Universidad de Ohio, a quien Rogers dirigió su tesis doctoral años atrás, y Rafael Obregón, publicaron una conversación sostenida con "Ev" en febrero de 2004, en la que hay múltiples elementos de coincidencia con el contenido de este texto (Singhal \& Obregón, 2005). 
sarrollo" editado por él, se incluyeron, entre otros, tres trabajos de especial importancia para la investigación latinoamericana.

Primero, Luis Ramiro Beltrán, en uno de sus textos más difundidos y citados (1976), analizó cómo y por qué "la investigación sobre comunicación en Latinoamérica ha estado, y todavía lo está, considerablemente dominada por modelos conceptuales foráneos, procedentes más que todo de Estados Unidos de América". Y se preguntaba:

Los críticos no han explicado la actitud pasiva e imitativa que se denuncia. ¿Se debe esto a pereza intelectual, a falta de competencia o a ambas? ¿Impide el entrenamiento de muchos investigadores latinoamericanos en Estados Unidos que éstos perciban su diferente realidad? ¿Reside quizá la respuesta en lo relativamente nuevo de la investigación en comunicación en Latinoamérica? ¿O constituyen la falta de perceptividad, de imaginación creadora y de audacia rasgos de una mentalidad conformista y acrítica que se somete, por definición, al colonialismo cultural? (Beltrán, 1976:77-78).

Antes de intentar dar respuestas, obviamente negativas, a estas preguntas con la propuesta de "Una nueva ciencia de la comunicación en Latinoamérica", Beltrán sintetizó bajo el subtítulo "Ceguera ante la estructura social", las principales críticas que los investigadores tanto estadounidenses como latinoamericanos habían hecho ya al difusionismo, desde sus mismos supuestos generales:

Un supuesto básico del enfoque de difusión es que la comunicación por sí misma puede generar desarrollo, independientemente de las condiciones socioeconómicas y políticas. Otro es que el incremento en la producción y consumo de bienes y servicios constituye la esencia del desarrollo y que, a su debido tiempo, se derivará necesariamente de ello una distribución justa del ingreso y de las oportunidades. Un tercer supuesto es que la clave del aumento en la productividad es la innovación tecnológica, sin tomar en cuenta a quiénes pueda beneficiar ni a quiénes pueda perjudicar (Beltrán, 1976:79).

Entre los análisis críticos que Beltrán recuperaba estaban los de Parra (1966), Havens y Adams (1966), Cuéllar y Gutiérrez (1971), Felstenhausen (1971), Díaz Bordenave (1974), Esman (1974) y el pro- 
pio Rogers (1975). Pero más allá de la investigación de la difusión de innovaciones, Beltrán cuestionó toda la tradición de origen estadounidense de estudios sobre la comunicación. En la sección dedicada a "La impronta de la teoría sobre el método", afirmaba que:

El modelo de Lasswell implica una concepción vertical, unidireccional y no procesual de la naturaleza de la comunicación. Definidamente, omite el contexto social. Al hacer de los efectos sobre el receptor la cuestión capital, concentra en él la atención de la investigación y favorece al comunicador como un poseedor incuestionado del poder de persuasión unilateral (Beltrán, 1976:91-92).

Esta preocupación original y fundante sobre los efectos de los mensajes y las funciones de los medios en la persuasión era para Beltrán la explicación del porqué "el análisis de contenido y la encuesta por muestreo, por vía de entrevistas estructuradas, llegaron a constituirse en el arsenal metodológico básico de la mayoría de los comunicólogos" (Beltrán, 1976:94). Pero la crítica de la pobreza teórica y la consecuente inadecuación para generar explicaciones pertinentes de la realidad social provino, antes que nada, de algunos investigadores estadounidenses y europeos (Nordenstreng, 1968; McLean, 1966), cuyos argumentos reforzaba Beltrán para concluir que:

Aunque gran parte de la investigación en comunicación realizada en Latinoamérica puede ser deficiente en cuanto a concepto y método, alguna es bastante refinada en cuanto al último y sigue muy de cerca los estándares norteamericanos, pero no es menos débil en conceptos. No pocos investigadores de la región parecen propensos a olvidar que la obsesión por las propiedades metodológicas puede conducir hacia "un énfasis indebido en la forma de conducir la investigación junto con un abandono de su sustancia" (Deutsch y Kraus, 1965:215). Habiendo aprendido a manejar bien los instrumentos matemáticos, algunas veces se enamoran tanto de ellos que el resultado que obtienen, a veces, es lo trivial o lo obvio empaquetado de manera impresionante en refinadas estadísticas. Algunos destacan, por ejemplo, la importancia de la confiabilidad al paso que soslayan la de la validez, lo cual puede producir deformaciones de la realidad social. Entonces 
se puede preguntar, como lo hace Halloran (1974:12): “¿Cuánto valor tiene ser preciso y consecuente respecto de algo que no es cierto o que no importa?" (Beltrán, 1976:103).

El trabajo de Beltrán concluía, como se había señalado, con la contrapartida a estas críticas y el señalamiento de lo que llevaba "Hacia una nueva ciencia de la comunicación en Latinoamérica":

En la mayoría de los casos, los nuevos investigadores de la comunicación han concentrado sus esfuerzos en la búsqueda de las ideologías de los comunicadores detrás de los contenidos manifiestos de sus mensajes en los medios masivos, tomando a éstos como expresiones de los intereses pro status quo de la estructura de poder que domina la sociedad. (...) La huella de la teoría también está naturalmente presente en el nuevo tipo de investigación. Por lo general, estos investigadores niegan a la refinación matemática de la metodología tradicional norteamericana el poder de llegar hasta los más profundos patrones de significado con los cuales están fundamentalmente preocupados (Verón, 1969; Mattelart, 1970). Por consiguiente, están recurriendo a técnicas no cuantitativas para análisis del mensaje o ensayando procedimientos semicuantitativos como accesorios a las percepciones intelectuales que procuran poner la investigación sobre comunicación al servicio del cambio estructural. (...) el hecho significativo es que, al fin, algunos estudiosos de la comunicación en Latinoamérica están dando señales de ser capaces de pensar por sí mismos y de enmarcar su trabajo en los términos de sus propias realidades (Beltrán, 1976:104-106).

El segundo de los trabajos latinoamericanos incluidos por Rogers entre las "Perspectivas críticas sobre comunicación y desarrollo" era el de Juan Díaz Bordenave (1976), sobre la necesidad de nuevos modelos para la comunicación de innovaciones agrícolas en América Latina. Su extensa revisión de lo que había sido la investigación comienza con un párrafo que, mediante una sencilla analogía, define el contenido:

Al igual que la ciencia aeronáutica evolucionó desde el concepto de motor lineal hasta la idea del motor de combustión circular, luego a la turbopropulsión y más recientemente hasta la era del motor a chorro, la ciencia 
de la comunicación también ha evolucionado desde el concepto lineal simple de información e influencia hasta una idea más compleja de la comunicación como componente social dinámico (Díaz Bordenave, 1976:135).

La crítica de las insuficiencias y desviaciones de la investigación de la comunicación para el desarrollo rural era retomada por Díaz Bordenave, igual que por Beltrán, de los investigadores estadounidenses y latinoamericanos mismos por una parte y, por otra, de la "revolución" iniciada por Paulo Freire (1973) al proponer "la abolición, en educación y comunicación, de la mentalidad de transmisión y el reemplazarla por una clase de comunicación educativa más liberadora, que contendría más diálogo y estaría al mismo tiempo más centrada en el receptor y más conciente de la estructura social" (Díaz Bordenave, 1976: 138). La necesidad de nuevos modelos para la investigación del desarrollo rural surgía de que:

En realidad, debido a que el modelo de difusión clásico se formuló bajo condiciones socio-económicas significativamente diferentes y de acuerdo con una posición ideológica incompatible con la realidad latinoamericana, las preguntas de investigación que utilizaron los investigadores latinoamericanos no tocan los puntos básicos que afectan el desarrollo rural (Díaz Bordenave, 1976:145).

Sobre preguntas más pertinentes a la realidad y a los intereses latinoamericanos, Díaz Bordenave proponía, como puntos focales prioritarios para la investigación en comunicación y desarrollo rural, los siguientes:

1 Estudiar la difusión y adopción como sistema de solución de problemas, comenzando no con la innovación y sus fuentes, sino con la situación, necesidades y problemas de los campesinos, considerando los flujos de comunicación horizontales y ascendentes por medio de los cuales los mediadores y los "centros de solución" articulan, transmiten y actúan sobre estas necesidades y problemas.

2 Estudiar el marco estructural en que se da (o no se da) la comunicación y la adopción, incluyendo la influencia de las formas de tenencia de la 
tierra, los efectos de la política agraria del gobierno sobre toma de decisiones y las consecuencias de la adopción de innovaciones dadas en cuanto al proceso global de desarrollo.

3 Estudiar los aspectos infraestructurales de la adopción de innovaciones, incluyendo sus relaciones configurativas con acceso a insumos, créditos, asistencia técnica, información, mercados, almacenaje, transporte y seguros.

4 Estudiar la adopción de innovaciones como experiencia de enseñanzaaprendizaje a fin de identificar las exigencias pedagógicas respecto a la difusión y adopción, como parte de un proceso más amplio de crecimiento mental y enriquecimiento humano de los campesinos.

5 Estudiar el funcionamiento de estrategias integradas de desarrollo rural en las cuales la concientización, educación, politización, organización y tecnificación desempeñen papeles integrados (Díaz Bordenave, 1976: 148-151).

Finalmente, en el tercero de los trabajos mencionados, el propio Everett Rogers (1976) retomaba y reformulaba muchas de las críticas y propuestas que hasta entonces se habían acumulado:

Los críticos de la pasada investigación de la comunicación señalan que, en ciertos aspectos, nuestra preocupación primaria por determinar los efectos en la audiencia a través de encuestas puede haber distraído nuestra atención científica de otros asuntos prioritarios como quiénes controlan los medios, cómo se toman decisiones sobre políticas y programación en esas instituciones, y cómo operan esas organizaciones para realizar sus funciones de mediación, procesamiento de la información, producción de mensajes y retroalimentación. Generalmente, estos críticos argumentan un giro del enfoque principal de la investigación en comunicación, de los efectos sobre la audiencia a la consideración de las instituciones de medios a través de un enfoque sistémico.

La comunicación para el desarrollo debe ser vista como un proceso total que incluye la comprensión del público y de sus necesidades, la planeación comunicativa alrededor de estrategias selectivas, la producción de mensajes, la diseminación, la recepción y la retroalimentación, más que 
sólo como una actividad unidireccional, directa, del comunicador al receptor pasivo. Esta conceptualización de la comunicación en el desarrollo implica un cuestionamiento del "enfoque de los componentes" en la investigación, frecuente en el pasado, en el cual se investigaba una variable de la fuente, una variable del mensaje o una variable del canal, para determinar cómo se relacionaba con uno o varios efectos. El enfoque de los componentes es esencialmente atomístico y mecanicista al desarticular heurísticamente los elementos del proceso comunicativo para tratar de entender su operación. Tal enfoque ignora la interacción sinérgica entre la fuente, el mensaje, el canal y el receptor. Falla al no captar la naturaleza sistémica del proceso comunicativo. Si la comunicación para el desarrollo se considera, entonces, como un proceso total, las interrelaciones entre sus componentes deben ser investigadas tanto como el entorno relevante en que el sistema de comunicación está inserto. Este acercamiento intelectual representa el enfoque sistémico de la comunicación para el desarrollo (Rogers, 1976:105).

Un texto de Enrique Sánchez Ruiz (1986) sirve muy bien para completar la revisión de los factores que pusieron en crisis el modelo comunicativo de la modernización, dando lugar no sólo a los enfoques de sistemas como los propuestos por Rogers, sino sobre todo a las corrientes críticas propiamente latinoamericanas:

Los problemas y anomalías de la tradición investigativa de la modernización por difusión hacen transparentes los problemas que enfrentaron los estudiosos sobre la comunicación, la educación y el desarrollo dentro de esta misma tradición. En los años sesenta y setenta, la evidencia comenzó a acumularse, mostrando que la característica imputada a los medios de comunicación como "multiplicadores mágicos" de la modernidad y del desarrollo no estaban correspondiendo a las expectativas (...).

Lo que todos estos estudios mostraban era que los programas de cambio social y de educación no formal para el cambio social, que hacían uso extensivo de los medios masivos -la mayoría de ellos implantados en entornos microsociales- no estaban logrando los resultados esperados de “desarrollo”. Por ejemplo, el estudio de Grunig (1971) entre campesinos 
colombianos mostraba que no todos los contenidos de los medios eran necesariamente "pro-desarrollo", como por ejemplo, presuponía Everett Rogers, cuya investigación era también sobre campesinos colombianos (Rogers y Svenning, 1969). Es decir, sólo la "información situacionalmente relevante", en particular con respecto a las necesidades de toma de decisión de los campesinos, podría producir una diferencia real en sus vidas. Pero, aun la información situacionalmente relevante fallaría en lograr algún cambio si existían rigideces estructurales, políticas y económicas, como la falta de acceso a la tierra, al crédito, a los insumos, etcétera. La conclusión de este tipo de estudios ha sido que "una comunicación habilidosa puede cambiar las percepciones de un campesino sobre su situación, pero no puede, actuando sola, cambiar mucho la situación. Puede ayudar a un granjero atrasado a ver oportunidades que él ignora, pero si existen pocas oportunidades, la información no las creará” (Brown y Kearl, 1967:25).

Entonces, concluían los investigadores, "el cambio estructural es la esencia del desarrollo y la comunicación un complemento" (Grunig, 1971: 582). Fueron muchos los estudios realizados durante la década pasada que apoyaban estas conclusiones básicas. El Instituto de Investigación de la Comunicación de la Universidad Stanford fue una vanguardia en el campo del estudio de la comunicación para el desarrollo durante las décadas de 1960 y 1970, bajo el liderazgo de Wilbur Schramm y con el apoyo financiero de diversas instituciones norteamericanas. Un reporte del mismo Instituto, que evaluaba quince años de actividades de la Agencia para el Desarrollo Internacional del Departamento de Estado norteamericano (US AID), llevaba como título La comunicación como complemento (Hornik et al, 1979) (Sánchez Ruiz, 1986:30-31).

En el informe de la evaluación dirigida por Hornik, resumida en un artículo publicado en 1980, que significó el término de los financiamientos otorgados por la AID al posgrado en Comunicación y Desarrollo de Stanford y a la mayor parte de los programas desarrollados durante las dos décadas anteriores en América Latina, pueden encontrarse los corolarios más importantes que los investigadores estadounidenses asociados al modelo difusionista de la modernización extrajeron de su amplia experiencia internacional: 
La comunicación para el desarrollo llena una docena de campos. Sus practicantes y sus investigadores han producido profusa y variadamente; una revisión de esos trabajos debe seleccionar sus fundamentos cuidadosamente, lanzar unas pocas preguntas y encontrar, si puede, un tema central para organizar las respuestas a esas preguntas.

Hemos puesto énfasis en aquellas aplicaciones que hacen algún uso de la tecnología comunicativa para proporcionar educación e información. Las tres preguntas que orientan esta revisión y sirven como guía son: ¿Qué roles desempeña la comunicación en el desarrollo? ¿Cuáles circunstancias llevan probablemente a una intervención particular al éxito o al fracaso? y finalmente, ¿qué sabemos sobre la promesa de aplicaciones específicas?

Un tema central resuena en todas las experiencias exitosas de los años recientes. La tecnología comunicativa funciona mejor como un complemento - a un llamado al cambio social-, a los cambiantes recursos, al buen diseño instruccional, a otros canales de comunicación, y a un conocimiento detallado de sus usuarios (Hornik, 1980:10).

El "réquiem por la modernización" (Sánchez Ruiz, 1986), fórmula que al Rogers de los años noventa le pareció adecuada y divertida, es finalmente una etapa importante de la historia de la investigación de la comunicación en América Latina ${ }^{9}$ y de la intervención de académicos estadounidenses en ella. Everett M. Rogers, uno de los más destacados entre ellos, pensaba que hay que seguir interpretando y aprendiendo de estas experiencias y de los desencuentros ideológicos que cruzaban y siguen cruzando el campo académico:

Yo tengo un punto de vista general, y no todos están de acuerdo con él, de que la diversidad en el pensamiento intelectual es una buena cosa y que no tenemos suficiente de esta diversidad en la mayor parte de los departamen-

${ }_{9}^{9}$ Pero que no ha sido "agotada" ni mucho menos como objeto de reflexión. Véase, como un excelente ejemplo de investigación académica reciente al respecto, el trabajo de Gustavo Cimadevilla (2004), premiado como la mejor tesis de doctorado en comunicación en Latinoamérica por la FELAFACS un año antes. 
tos o escuelas de comunicación, al menos en Estados Unidos. Pero también reconozco que si tienes demasiada diversidad, la gente no se entiende y no quiere entenderse mutuamente. Y resultan islas separadas dentro de la misma estructura organizacional. Pero en general, pienso que la mayoría de los departamentos universitarios de comunicación de Estados Unidos necesita mayor diversidad y me alegra cuando esto ocurre.

\section{BIBLIOGRAFÍA}

ANZOLA, Patricia y Patricio Cooper (1985) La investigación en comunicación social en Colombia. Lima: DESCO.

ATWOOD, Rita (1980) "Communication research in Latin America. Cultural and conceptual dilemmas." Paper presented to the Intercultural Division, International Communication Association Convention, Acapulco.

BELTRÁN S., Luis Ramiro (1976) "Alien premises, objects and methods in Latin American communication research." Communication Research, an international quarterly, vol. 3 núm. 2, p.107-134. En español: "Premisas, objetos y métodos foráneos en la investigación sobre comunicación en América Latina", en Moragas (ed.), Sociología de la comunicación de masas. I: Escuelas y autores. Barcelona: Gustavo Gili, 1985. p.73-107.

BELTRÁN S., Luis Ramiro, Guillermo Isaza y Fernando Ramírez (1976) Bibliografía sobre investigaciones en comunicación para el desarrollo rural en América Latina. Bogotá: CIID.

BROWN, M. R. y B. E. Kearl (1967) Mass communication and development: The problem of local and functional relevance. Madison: University of Wisconsin.

CANO Gallego, Jairo (1971) Un sistema de información para elevar la productividad agrícola; conceptualización y análisis del beneficio/costo del Plan Puebla. Tesis de Maestría en Ciencias, Chapingo: Colegio de Posgraduados.

Cimadevilla, Gustavo (2004) Dominios. Crítica a la razón intervencionista, la comunicación y el desarrollo sustentable. Buenos Aires: Prometeo Libros.

CUÉLlAR G., David y Jaime Gutiérrez S. (1971) “Análisis de la investigación y de la aplicación del difusionismo". Ponencia en la Segunda Reunión de Comunicadores Rurales, Cali, Colombia, octubre.

DeUTSCH, M. y R. M. Kraus (1965) Theories in Social Psychology. New York: Basic Books.

Deutschmann, Paul J. y Orlando Fals Borda (1962) Communication and adoption patterns in an Andean village. San José, Costa Rica: Programa Interamericano de Información Popular.

DíAz Bordenave, Juan (1964) "Bonito and Timbauba: exploratory study of the leaders of two towns of the Brazilian Northeast, at different levels of development." Paper presented to the American Society for Applied Anthropology, San Juan Puerto Rico. 
- (1966) The search for instrumental information among farmers of the Brazilian Northeast. PhD Thesis, East Lansing: Michigan State University.

- (1972) "New approaches to communication training for developing countries." Paper presented at the Third World Congress of Rural Sociology, Baton Rouge Louisiana. En español: "Nuevos métodos de entrenamiento de la comunicación para los países en desarrollo” en Chasqui (primera época, 7). Quito: CIESPAL, 1974.

- (1974) "Communication and adoption of agricultural innovations in Latin America." Paper presented to the International Symposium on Communication Strategies for Rural Development, New York: Cornell University.

DíAZ Cisneros, Heliodoro y Herman Felstenhausen (1972) "Communication and institutional change in Mexican agricultural development." Paper presented at the Third World Congress for Rural Sociology, Baton Rouge Louisiana, August.

ESMAN, M. (1974) "Popular participation and feedback systems in rural development." Paper presented at Cornell-CIAT International Symposium on Communication Strategies for Rural Development, Ithaca, NY: Cornell University.

FELSTENHAUSEN, Herman (1971) "Conceptual limits of development communications theory." Paper presented at the Association for Education in Journalism, Columbia, South Carolina.

FREIRE, Paulo (1973) ¿Extensión o comunicación? la concientización en el medio rural. México: Siglo XXI.

FUENTES Navarro, Raúl (1992) Un campo cargado de futuro. El estudio de la comunicación en América Latina. México: FELAFACS.

GRUNIG, James E. (1971) "Communication and the economic decision-making process of Colombian peasants." Economic Development and Cultural Change, July, p.580-597.

HALLORAN, James D. (1974) Mass media and society: The challenge of research. Leicester UK: Leicester, University Press.

HAVENS, A. F. y D. W. Adams (1966) "The use of socio-economic research in developing a strategy of change in rural communities: a Colombian example." Economic Development and Cultural Change (14, 2). p.204-216.

HORNIK, Robert et al (1979) Communication as complement: An overview of communication in development. Institute of Communication Research, Stanford University.

- (1980) "Communication as complement in development." Journal of Communication, vol.30, núm. 2 (Spring), p.10-24.

JIMÉNEZ Sánchez, Leobardo (1963) Adoption/non-adoption of agricultural innovations by farmers in some areas of the State of Mexico. MS Thesis, Madison: University of Wisconsin.

- (1967) Socio-economic change and communication; a study of development of a farming ejido in central Veracruz, Mexico. PhD Thesis, Madison: University of Wisconsin. 
KLAPPER, Joseph (1960) The effects of mass communication. Glenco, Illinois: The Free Press.

KRIPPENDORFF, Sultana (1979) "The communication approach to development: A critical review", Studies in Third World Societies (Third World Mass Media: Issues, Theory and Research) (9, september).

LASSWELL, Harold D. (1985) "Estructura y función de la comunicación en la sociedad." en Moragas (ed), Sociología de la Comunicación de Masas. II Estructura, Funciones y Efectos. Barcelona: Gustavo Gili. p. 50-68.

LERNER, Daniel (1958) The passing of traditional society: Modernizing the Middle East. Glencoe Illinois: The Free Press.

— (1963) "Toward a communication theory of development" in L. W. PYE (ed.) Communications and political development. Princeton NJ: Princeton University Press.

MARQUES De Melo, José (Org.) (1978) Comunicação, modernização e difusão de inovacões no Brasil. Petrópolis RJ: Vozes.

- (1984) "La investigación latinoamericana en comunicación", Chasqui No. 11, Quito: CIESPAL, p. 4-11.

MARTÍNEZ Reding, Jesús (1963) La difusión y adopción del maíz híbrido en cuatro municipios del estado de Guanajuato. Tesis, Chapingo: Colegio de Posgraduados.

- (1965) Study of the behavior of three frequency divisions of words in the inventory of Spanish vocabulary. MS Thesis, Madison: University of Wisconsin.

MARTÍNEZ Valdés, Gregorio (1960) La página agrícola del periódico El Dictamen de Veracruz: su lectoría y lectura. Tesis de Ingeniero Agrónomo, Saltillo: Escuela Superior de Agricultura Antonio Narro.

- (1962) Developing and testing a new type of bulletin for use in Mexican agricultural information program. MS thesis, Madison: University of Wisconsin.

- (1970) Comprehension of pictorial messages on corn production by literate, semi-literate and illiterate farmers in central Veracruz, Mexico. $\mathrm{PhD}$ Thesis, Madison: University of Wisconsin.

MATTELART, Armand (1970) "Críticas a la Communications Research." Cuadernos de la Realidad Nacional, Edición Especial, 3. Santiago de Chile, p. 11-22.

MCLEAN, M. S. Jr. (1966) "Frontiers of communication research" in: Procceedings, Convention of Journalism Institutes, Madison: University of Wisconsin.

MORAGAS, Miquel de (1981) Teorías de la comunicación. Investigaciones sobre medios en América y en Europa. Barcelona: Gustavo Gili.

MURGA, Antonio y Guillermo Bolis (1979) "Sociedad y ciencia social en Latinoamérica", en Bolis y Murga (eds). Las ciencias sociales en América Latina. México: UNAM, p. 9-31. 
MYREN, Delbert T. (1974) "Analysis of communication in the Puebla project." In: International Conference on Integrated Communication for Rural Development. Honolulu, Hawaii: East-West Center, p.129-131.

NORDENSTRENG, Kaarle (1968) "Communication research in the United States: a critical perspective." Gazette $(14,3)$.

PARRA, S. R. (1966) La estructura social y el cambio de la tecnología: el caso de Candelaria. Bogotá: Universidad Nacional.

RoGERS, Everett M. (1962) Diffusion of Innovations. New York: The Free Press of Glencoe.

- (1964) "Diffusion of innovations in rural societies." Research proposal to the Agency for International Development, East Lansing, Michigan: Michigan State University.

— (1973 Communication strategies for family planning. New York: The Free Press.

- (1975) "Where we are in understanding the diffusion of innovations." In: Schramm and Lerner (eds.), Communication and change in the developing countries: Ten years after. Honolulu, Hawaii: East-West Center.

- (1976) "Communication and development: the passing of the dominant paradigm”, Communication Research, an international quarterly, vol. 3 núm. 2.

- (1994) A history of communication study. A biographical approach. New York: The Free Press.

— y Elssy Bonilla De Ramos (1965) "Prediction of the adoption of innovations: a progress report." Paper presented at the Rural Sociological Society, Chicago, August.

- y William Herzog (1966) "Functional literacy among Colombian peasants" Economic Development and Cultural Change, núm. 14, p.190-203.

- y L. Svenning (1969) Modernizating peasants: The impact of communication. New York: Holt, Rinehart and Winston.

Rostow, W.W. (1960) The stages of economic growth. Cambridge, UK: Cambridge University Press.

RUANOVA Hernández, Alfonso (1956) La exposición a ferias agrícolas como un método de divulgación a los agricultores. Tesis de Ingeniero Agrónomo, Saltillo: Escuela Superior de Agricultura Antonio Narro.

- (1958) Content and readability of some Latin American agricultural magazines. MS Thesis, Madison: University of Wisconsin.

SÁNCHEZ Ruiz, Enrique E. (1986) Réquiem por la modernización: perspectivas cambiantes en estudios del desarrollo. Guadalajara: Universidad de Guadalajara (Cuadernos de Difusión Científica, núm. 7).

SAPERAS, Enric (1985) La sociología de la comunicación de masas en los Estados Unidos. Una introducción crítica. Barcelona: Ariel.

SCHRAMM, Wilbur (1961) La ciencia de la comunicación humana. México: Roble.

- (1964) Mass media and national development. Stanford: Stanford University Press. 
- (1965) Desarrollo de la comunicación y desarrollo económico. San José, Costa Rica: Instituto Interamericano de Ciencias Agrícolas de la OEA (Publicación Miscelánea, núm. 25, mimeo).

- (1997) The beginnings of communication study in America. A personal memoir (edited by Steven H. Chaffee and Everett M. Rogers). Thousand Oaks, California: Sage.

SINGHAL, Arvind y Rafael Obregón (2005) “A conversation with Everett Rogers (february 2004)", Consortium Dialogues. [http://www.communicationforsocialchage.org, consultado 25/02/2005].

VERÓN Eliseo (1969) "Ideología y comunicación de masas: la semantización de la violencia política”, en Verón (ed.), Lenguaje y comunicación social. Buenos Aires: Nueva Visión, p. 133-190. 